\title{
Postevent cues bias recognition performance in pigeons
}

\author{
DAVID N. HARPER and MARYANNE GARRY \\ Victoria University, Wellington, New Zealand
}

\begin{abstract}
In the present study, we examined whether the presentation of postevent cues would bias recognition in a visual delayed matching-to-sample task with pigeons. Postevent cues were either consistent with the original target stimulus (i.e., they were the same as the correct choice option at recognition), inconsistent (i.e., they were the same as the incorrect recognition option), or neutral (i.e., they were different from both the correct and the incorrect recognition options). In Experiment 1, a single colored light served as the target stimulus. In Experiment 2, the target stimulus was one of three lights presented in a sequence. Both experiments demonstrated that recognition choices were biased toward the option corresponding to the postevent cue, but only if the cue occurred at the end of the delay interval. The present results mirror those found using the misinformation paradigm with human subjects.
\end{abstract}

The delayed matching-to-sample (DMTS) task is an established procedure for examining working memory performance in nonhuman subjects. A typical DMTS trial involves presentation of a target sample stimulus followed by a delay of variable duration, during which the stimulus is not available. At the end of the delay, the subject is required to recognize or recall the target stimulus when given a choice between two or more alternatives. In studies using pigeons as subjects, the stimuli have typically been colored keylights, lines, or forms, and the delays have varied between 0 and $40 \mathrm{sec}$. Such procedures have been used to explore the effects of many of the variables known to influence the memory function, including those of reinforcement (e.g., McCarthy \& Voss, 1995; Nevin \& Grosch, 1990), of brain lesions and drugs (e.g., Aggleton, Keith, \& Sahgal, 1991; Harper, McLean, \& Dalrymple-Alford, 1994; Kirk, White, \& McNaughton, 1988), and of various sources of interference (e.g., Edhouse \& White, 1988; Harper \& White, 1997; Jans \& Catania, 1980; Maki, Moe, \& Bierley, 1977; Roberts \& Grant, 1978; White, 1985).

With respect to interference effects in DMTS tasks, researchers have examined the influence of events occurring prior to the current trial (proactive interference) and during the delay interval of the current trial (retroactive interference) on recognition performance (Edhouse \& White, 1988; White, 1985). Studies that have explored these two types of interference have not only differed with respect to the location of the interfering stimulus, but also with respect to the nature of the extraneous stimuli. In the

\footnotetext{
The research was supported by grants from the Victoria University of Wellington Internal Grants Committee. We thank the staff and students who helped conduct this research in the VUW School of Psychology Animal Facility. We also appreciate the useful comments made by Keith Horton on an earlier version of this article. Correspondence concerning this article should be addressed to D. N. Harper, School of Psychology, Victoria University of Wellington, P.O. Box 600, Wellington, New Zealand (e-mail: david.harper@vuw.ac.nz).
}

proactive interference research, the stimuli are the same as, or closely related to, the stimuli that the subject is required to discriminate when given a recognition choice. These studies have shown that subjects are biased toward a response alternative in the current trial that corresponds to the correct alternative in the immediately preceding trial (Edhouse \& White, 1988). For example, if the correct target stimulus in the immediately previous trial was a red light, a subject would be biased toward choosing the red alternative on the current trial, irrespective of whether red or green was the correct target for the current trial.

In contrast, the focus of the retroactive interference research has been on the effect of novel, and therefore disruptive, stimuli that impair (as opposed to bias) performance. For example, a number of studies with pigeons have shown that accuracy is impaired when a houselight is illuminated during a normally dark delay interval (Harper \& White, 1997; Maki et al., 1977; Roberts \& Grant, 1978; White, 1985) or when the food hopper is operated during the delay (Jans \& Catania, 1980). However, although previous research has demonstrated that stimuli presented during the delay can impair accuracy, very little is known about the degree to which DMTS performance can be biased toward making one choice in favor of another as a result of stimuli presented during the delay.

The issue of whether DMTS performance in animal subjects can be biased by stimuli occurring during the delay interval is interesting and relevant, given the attention that this issue has received in the human memory literature from studies employing the misinformation paradigm (Belli, 1989). This paradigm is similar to the DMTS procedure used to explore retroactive interference effects in animals, except that the human paradigm presents postevent cues during the delay that bias subsequent recognition, rather than merely lowering overall accuracy. That is, some of the cues presented during the delay interval actually improve accuracy relative to a baseline level (by providing the subject with information that is 
consistent with the original event), but other cues presented during the delay lower accuracy relative to a baseline level (by providing the subject with information that is inconsistent with the original event).

One of the early studies to employ the misinformation paradigm was the now classic work of Loftus, Miller, and Burns (1978). In their study, subjects were shown a series of slides depicting a road accident. After a delay, subjects answered a questionnaire that contained either consistent or misleading information. For example, subjects might read, "Did another car pass the red Datsun while it was stopped at the stop sign?" For those subjects who did in fact see a stop sign in the series of slides, this question contained consistent information. For those subjects who had actually seen a yield sign in the series of slides, this question contained misleading information. Loftus et al. found that a subject's subsequent recognition of events depicted in the slides was influenced by the content of the information provided in the questionnaire. For example, subjects who were given misleading information (were told that there was a yield sign when, in fact, a stop sign had been shown in the slides) performed worse than chance. By contrast, subjects who were provided with information consistent with the original event (were told that there was a stop sign) performed more accurately than subjects given no postevent information. This effect of postevent cues on recognition has been called the misinformation effect (Belli, 1989).

In the present study, we examined whether such an effect could be demonstrated in an analogous task using pigeons as subjects. In addition to determining whether a basic misinformation effect could be demonstrated in a nonhuman species, in the present study, we examined whether the postevent cue interferes with memory for the original target stimulus or whether the postevent cue serves to bias recognition independent of memory for the original stimulus. With respect to this issue, McCloskey and Zaragoza (1985) and Belli (1989) have argued that an informative condition in the human misinformation paradigm is to present subjects with a potentially relevant postevent cue (as opposed to no postevent cue at all) but then not offer that cue as an alternative at recognition. For example, McCloskey and Zaragoza showed subjects a slide sequence depicting the illegal actions of a burglar posing as a repairman. In one condition, the burglar used a hammer to hide a stolen object, but misled subjects were told that the tool was a screwdriver. During a recognition test, some subjects were given a standard Loftus-type test and were asked to choose between the original stimulus (hammer) and the postevent stimulus (screwdriver); other subjects were given a modified test and were asked to choose between the hammer and a completely novel stimulus (in this case, a wrench). McCloskey and Zaragoza looked for differing patterns of misinformation effects between subjects tested under standard and modified conditions. More specifically, they looked to see whether subjects under standard testing conditions were less likely to choose the hammer but subjects in the modified condition were equally likely to choose the hammer. Mc-
Closkey and Zaragoza found a misinformation effect the standard Loftus-type test, but no such effect on $t$ modified test. They concluded that the postevent cue do not serve to interfere with the memory of the original $t_{\mathrm{t}}$ get stimulus; rather, the postevent cue serves to bi recognition independent of how well the subject remei bers the original target stimulus.

\section{EXPERIMENT 1}

In Experiment 1, we examined whether a misinform tion effect could be demonstrated with pigeons as su jects. The DMTS procedure was adapted in a manner th was similar to the way in which McCloskey and Zarago (1985) adapted Loftus et al.'s (1978) task. That is, i stead of presenting no or totally irrelevant information a neutral condition, the postevent cues used on such tric were potentially relevant, in that they were also used target stimuli on other trials. Across different trials, thr different colors served as target stimulus, postevent $\mathrm{cu}$ and choice options. By using the colored lights inte changeably in this way, a neutral trial might take one several forms. For example, a red stimulus might be $\mathrm{fc}$ lowed by a green light (as a neutral postevent cue), and the recognition phase, the subject would be given a choi between red and yellow lights. Or, another neutral tri might involve a green stimulus light followed by a yello postevent cue, with a subsequent choice between gret and red light options. In all cases, as with the procedu used by McCloskey and Zaragoza, the neutral cue is $p$ tentially relevant (and is, therefore, potentially able to $\mathrm{i}$ terfere with memory) but is not offered as a choice optic at recognition.

Initially, pigeons were trained in the basic DMTS ta: in order to establish baseline levels of accuracy when $r$ postevent cue was presented after the original targ stimulus. That is, the subjects were presented with a re green, or yellow target stimulus, which they had to re ognize after a variable delay of between 2.5 and 20.0 se Subsequently, the effects of postevent cues were assess across two conditions. In one condition, the subjects we: exposed to three different types of trials by presentir different postevent cues at the end of the delay interva On a consistent trial, the postevent cue was the same : the original target stimulus. On an inconsistent trial, th postevent cue was different from the target stimulus b the same as the incorrect choice option at recognition. C a neutral trial, the postevent cue was different from bol the correct target stimulus and the incorrect choice optio] If performance follows the same pattern as that in th misinformation effect identified in humans, accurac should be higher on consistent trials and lower on incor sistent trials, and there should be no difference in accurac between neutral trials and trials in which no postevent $\mathrm{cl}$ is presented at all.

In the other condition, the types of trials remained th same, but the location of the postevent cue was move from the end of the delay interval to the beginning of th delay interval (i.e., immediately after target stimulus prs 
sentation). Previous research, in which retroactive interference effects in DMTS tasks (Roberts \& Grant, 1978) and misinformation effect research in humans (Belli, Windschitl, McCarthy, \& Winfrey, 1992; Loftus et al., 1978) were examined, indicated that the magnitude of the recognition bias caused by the postevent cue depended on when, during the delay interval, the cue or the disruptive event was presented. Cues that were presented relatively closer to original stimulus presentation than to the recognition test had less of an effect on accuracy than did cues that were closer to recognition than to the original stimulus presentation. In this fashion, we examined whether the misinformation effect of postevent cues at the end of the delay interval was attenuated by presenting the postevent cues at the start of the delay.

\section{Method}

Subjects. Six individually housed adult homing pigeons, with extensive prior experience in a DMTS procedure that used the same stimuli as those used here, were maintained at $85 \%$ of their freefeeding body weights. Supplementary feed was given after the final session of the day to maintain prescribed body weights. The housing room was maintained at a constant $22^{\prime \prime} \mathrm{C}$, with lights on between 7.00 a.m. and 7.00 p.m.

Apparatus. A sound-attenuating response chamber, measuring $29 \times 25 \times 29 \mathrm{~cm}$, contained an interface panel with a centrally mounted grain hopper and three response keys. One response key was mounted directly above the hopper, and the other two keys were mounted above and to either side of the hopper (with a distance of $8 \mathrm{~cm}$ between the centers of adjacent keys). Each key could be illuminated red, green, blue, white, or yellow from behind. All the experimental events were scheduled and recorded by an IBMcompatible computer running MED-PC software.

Procedure. Prior to conducting the experimental sessions, pigeons received 30 sessions of training on the basic DMTS task. Each trial began with the central key being lit from behind by a red, green, or yellow light. The fifth peck to this key turned the light off and initiated a delay $(2.5,7.5$, or $20.0 \mathrm{sec})$. During the delay, the chamber was dark, and responses were ineffective. The order of the sample lights was randomized across trials, with the constraint that the same sample could not occur on more than four successive trials. Delay intervals were also randomized across trials, with the constraint that a particular delay was not repeated until the other delays had occurred for a given stimulus. The delay interval terminated with the onset of the two side key comparison stimuli. The side keys were each lit a different color from the range of colors used as sample stimuli. That is, the side keys were lit using redgreen, red-yellow, or green-yellow combinations, with the choice of which color would go on the left key and on the right key randomized across trials. The two side keys remained illuminated until the pigeon pecked once at one of the keys. A correct choice was defined as a peck to the side key color that matched the sample stimulus. Correct responses darkened both keys and produced a $2.5-\mathrm{sec}$ access to the reinforcer, followed by a darkened 20 -sec intertrial interval. Incorrect responses darkened both keys and produced a 2.5 sec time-out, followed by a 20 -sec intertrial interval. The data from the last 7 days of training were used to assess baseline performance for each pigeon in the present task when no postevent cues were presented in the delay interval (the no-cue condition).

After training in the basic DMTS task, the effects of extraneous postevent stimuli presented during the delay were assessed across two conditions, with each condition lasting 60 sessions. Each pigeon received both conditions, with the order of conditions reversed for half the subjects. In one condition, the postevent cue was the central sample key, illuminated red, green, or yellow after the delay period had timed out (i.e., at the end of the delay period). A single peck to the key turned off the central light and turned on the two side comparison lights. In the other condition, the postevent cue was the central sample key, illuminated red, green, or yellow immediately after the fifth peck at the initial sample stimulus light (i.e., at the beginning of the delay period). A single peck to the key turned off the central light and initiated the delay before the two side keys were illuminated. One pigeon stopped responding during this condition, and its data are not included in the final analysis.

Trials were categorized in one of the following three ways when a postevent cue was presented. If the postevent cue was the same as the sample stimulus (i.e., red sample stimulus followed by a red postevent cue, green sample followed by green cue, or yellow sample followed by yellow cue), the trial was classified as a consistent trial. For these trials, the postevent cue was, therefore, also the same as the correct choice option at the end of the trial. If the postevent cue was different from the sample stimulus but was the same as the incorrect choice option, the trial was classified as an inconsistent trial-for example, a red sample stimulus light followed by a green postevent cue followed by a choice between red and green, or a yellow stimulus light followed by a red postevent cue followed by a choice between red and yellow, and so forth. Finally, if the postevent cue was different from the sample stimulus but was also different from either choice option, the trial was classified as a neutral trialfor example, a red sample stimulus light followed by a green postevent cue followed by a choice between red and yellow, or a yellow stimulus light followed by a red postevent cue followed by a choice between green and yellow, and so forth. Approximately equal numbers of consistent, inconsistent, and neutral trials were conducted per session in a randomized order, with the constraint that no more than four trials in a row were of the same type.

In all phases of Experiment 1, experimental sessions were conducted 7 days per week and comprised 84 trials or lasted $50 \mathrm{~min}$, whichever occurred first.

\section{Results and Discussion}

The data used for the present analysis were percentage of correct responses made over the last 40 sessions of each condition, separated according to trial type (consistent, inconsistent, or neutral), and over the last 7 sessions of baseline performance, when no cue was presented during the delay. The rejection level adopted for all statistics subsequently performed here and in Experiment 2 was $p<.05$.

The top graph in Figure 1 shows the mean percent correct (averaged over all the subjects) at each delay for consistent, inconsistent, and neutral trials for the condition in which the postevent cue was presented at the start of the delay. On all trial types, there was a general monotonic decrease in accuracy as delay increased. Accuracy, however, did not change as a product of trial type. Collapsed across delays, the overall average percent correct was $69 \%, 65 \%$, and $67 \%$ for consistent, inconsistent, and neutral trial types, respectively. The consistency of the patterns shown in Figure 1 across individual subjects is supported by a repeated measures analysis of variance (ANOVA) comparing trial type and delay. This analysis revealed a significant main effect of delay on accuracy $[F(2,8)=19.4]$, but no effect of trial type.

The bottom graph in Figure 1 shows the mean percent correct (averaged over all the subjects) at each delay for consistent, inconsistent, and neutral trials for the condition in which the postevent cue was presented at the end 

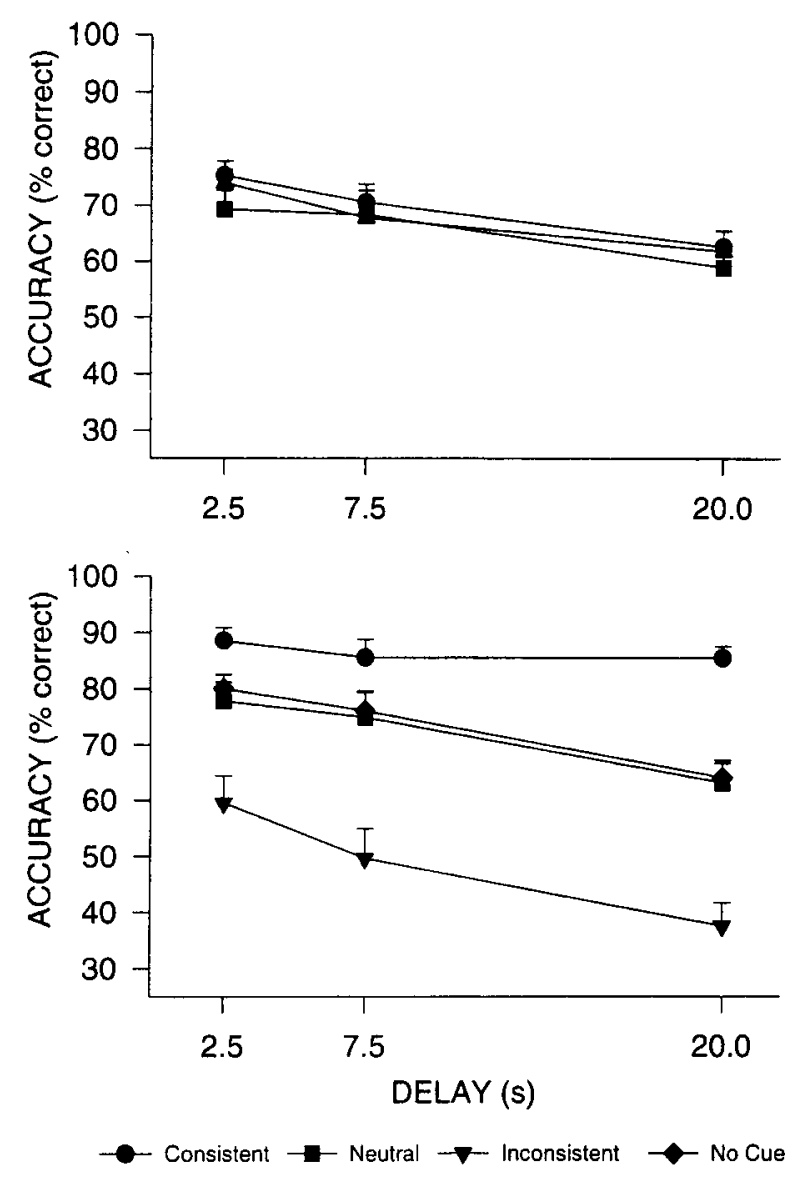

Figure 1. Mean percent correct (averaged across subjects) as a function of delay when the postevent cue was presented at the start of the delay interval (top graph) and at the end of the delay (bottom graph) in Experiment 1. Data are presented separately for consistent (circles), neutral (squares), inconsistent (triangles), and no-cue (diamonds) trial types. Error bars show the standard error associated with each data point.

of the delay. Included in this graph are the data from the baseline condition in which no cue was presented during the delay. This graph indicates that there were two main trends in the data. First, regardless of trial type, there was a decrease in accuracy as the delay increased. Second, accuracy was greater overall when trials involved postevent stimuli that were consistent with the target sample stimulus. On the other hand, accuracy was poorer overall when trials involved postevent stimuli that were misleading. Performance did not differ between the neutral condition (in which the postevent stimulus was different from both the correct and the incorrect choice options) and that in which no cue was presented in the delay at all. The systematic nature of these patterns across all the subjects is supported by the results of repeated measures ANOVAs comparing trial type and delay. This analysis revealed a significant main effect of trial type $[F(3,15)=17.0]$ and delay $[F(2,10)=150.6]$ on accuracy. Pairwise comparisons of the different trial types indicated that accuracy was lower on the neutral trials than on the consistent trials
$[F(1,5)=8.7]$, that accuracy was higher on the neutr trials than on the inconsistent trials $[F(1,5)=23.3]$, b that there was no difference in accuracy on the neutral $t$ als, as compared with the no-cue trials $[F(1,5)=1.6]$. $\mathrm{T}]$ overall analysis also revealed the existence of an inte action between trial type and delay $[F(6,30)=32.4]$. Pai wise comparisons of the conditions at each delay indicat that this interaction arose because, at the shortest dela there was no significant difference in performance acro different types of trial $[F(3,20)=1.4]$ but that, at each the remaining delays, there was a significant differen according to trial type $[F(3,20)=12.8$ and $F(3,20)=4$. for 7.5- and 20.0-sec delays, respectively]. Therefore, $c$ though placing a postevent cue at the end of the delay $i$ terval biased recognition, the effect was more pronounct at longer delays, relative to the shortest delay.

In summary, the results of Experiment 1 demonstrat; that pigeons in a DMTS task produced patterns consiste with those observed in human misinformation studie These results mirror the original results of Loftus et a (1978) in several ways. When a postevent cue is presents at the end of the delay interval and immediately prior recognition, responses are biased toward the choice co responding to the postevent cue (as opposed to the targ stimulus). Consequently, when the postevent cue is co sistent with the target sample stimulus, accuracy is great than when the postevent cue is neutral, inconsistent, or a sent. When the postevent cue is misleading, subjects a biased toward making the wrong choice and, thus, pe form worse than when the cue is neutral or absent. Al: consistent with the previous human data (Belli et a 1992; Loftus et al., 1978) was the observation that $t$ extent to which the postevent cue biased recognition w: a product of when, during the delay, it occurred and $t$ length of the delay interval. Specifically, there was no e fect of a postevent cue when it occurred at the start of $\mathrm{tl}$ delay interval. Likewise, the biasing effect of the posteve cue was relatively smaller following short delay inte vals, as compared with longer intervals, especially if $t \mathrm{l}$ cue contained information consistent with the origin stimulus event.

The attenuating effects of delay length and cue locatic within the delay might result from several factors. For $\mathrm{e}$ : ample, at short delays, subjects tend to be relatively acc rate; thus, it is unlikely that they can benefit to the san degree from the facilitating effects of consistent info mation on short-delay trials, as compared with long-deli trials. Similarly, the likelihood of being biased by th postevent cue may rely not only on a decay in the memo: for the original target stimulus, but also on the ability remember the postevent cue. Therefore, the closer the $\mathrm{cr}$ is to recognition versus initial stimulus presentation, th more likely the subject is to recognize the cue, as compare with the target stimulus. Finally, previous pigeon DMT research in which the disruptive effect of illuminating novel bright light during the delay was examined $h_{i}$ shown that the later in the delay interval the light is illı minated, the greater the impairment in accuracy (Rober \& Grant, 1978). 
Assuming the equivalence of the present task with those used previously, Experiment 1 replicated the basic misinformation effect. In addition, this experiment replicated the findings of more recent studies that have attempted to clarify the mechanisms through which the postevent cue comes to influence performance. McCloskey and Zaragoza (1985) and Belli (1989) demonstrated that recognition performance was the same if the postevent cue contained neutral information (information that was dissimilar to both the correct and the incorrect recognition options), as compared with trials in which no postevent cue was presented at all. On the basis of this observation, they concluded that postevent cues do not act by interfering with the memory for the original target stimulus. Because we observed the same pattern of results as McCloskey and Zaragoza (see neutral vs. no-cue conditions in the bottom graph of Figure 1), we conclude that the postevent cues acted in the same fashion here. That is, the postevent cue appears to bias recognition choices, not because it interferes with memory for the original sample stimulus, but because of confusion or an acceptance of the cue as representing the correct choice option. Put another way, pigeons can demonstrate misinformation acceptance.

\section{EXPERIMENT 2}

In Experiment 2, we examined whether the patterns of effect obtained in Experiment 1 persisted when the identity of the target stimulus was not explicit. Although the pattern of results found in Experiment 1 mirrored those that would be expected if the present task was analogous to the human misinformation paradigm, there are still a number of procedural differences between the human and the nonhuman tasks. One of the major differences is the degree to which the target stimulus is made explicit to subjects at the start of a trial. In the human paradigm, subjects are not aware which particular event they may be subsequently asked to recall or recognize. For example, the target stimulus may be just one event that occurs in a sequence of slides. The procedure used in Experiment 1 presented target stimuli in the standard manner normally employed in the majority of DMTS tasks with rats, monkeys, and pigeons. That is, a single discrete stimulus was presented as the target. Such a method of stimulus presentation makes the target very explicit; there is only one possible correct option in a given trial. Such a situation allows subjects to engage in potentially very effective mediating behaviors to aid performance. For example, some studies, using rats as subjects and lever position as stimuli, have required the subject to respond on an extraneous lever located elsewhere, in order to prevent their standing beside the correct lever throughout the delay interval (e.g., Harper et al., 1994). In comparison, because the human misinformation task does not make the target stimulus explicit, subjects are unlikely to engage in rehearsal strategies that may confound the effectiveness of postevent cues. Consequently, the standard method of delivering a single target stimulus to a subject on each trial represents a major procedural difference between the DMTS task used in Experiment 1 and the human misinformation paradigm.

In Experiment 2, the target stimulus was made less explicit by presenting subjects with a sequence of three colors at the start of each trial, only one of which was subsequently tested for recognition. The aim was to replicate the patterns of effect caused by postevent cues dependent on cue type (consistent, inconsistent, neutral, or no-cue) and location (beginning vs. end of the delay) shown in Experiment 1, using a procedure that reduced the possibility of confounding mediating behaviors.

\section{Method}

Subjects and Apparatus. The subjects and apparatus were the same as those used in Experiment 1. Note that, between Experiments 1 and 2 , the pigeons received approximately 6 months of training in an earlier version of the procedure eventually used in Experiment 2. Thus, prior to Experiment 2, pigeons had received extensive experience in a DMTS task employing the same stimuli, and overall performance was consistently above chance levels.

Procedure. The procedure used in Experiment 2 was the same as that in Experiment 1, except for the following differences: First, the target stimulus was one of three colored lights presented in a sequence at the start of each trial. For example, at the start of a trial, the central key might be lit red, followed by green and then, finally, yellow. To progress from one color to the next (and then to initiate the delay after presentation of the third color), pigeons were required to peck at each color three times. The delay began after the stimulus list had been presented. After the delay, the two side keys were illuminated, with the target stimulus (one of the three lights presented at the start of the trial) on one key and an incorrect option (not one of the three lights presented at the start of the trial) on the other key. As in Experiment 1, the pigeons were required to peck once at the side key that was illuminated the same color as the original target stimulus in order to gain reinforcement. Whether the correct target stimulus was the first, second, or third color in the list was randomly varied over trials.

As in Experiment 1, trials were classified as one of four different types: consistent, inconsistent, neutral, or no-cue, depending on the nature of the postevent cue presented on the central key during the delay (see Experiment 1 for definitions and examples of the trial types). In Experiment 2, however, all four trial types were presented within any given session (as opposed to Experiment 1, where nocue trials were conducted separately from the other trials). Also, as in Experiment 1, the location of the postevent cue was varied across two conditions in Experiment 2. In one condition, the postevent cue was presented as soon as the delay interval had timed out, and in the other condition, the postevent cue was presented at the start of the delay. In both conditions, a single peck at the central key turned the postevent cue off. Across trials, red, green, yellow, blue, and white were all used for the target stimuli, postevent cue, correct response option, and incorrect response option. In Experiment 1, these two conditions were run sequentially for 60 sessions each. However, in Experiment 2, whether the postevent cue occurred at the start or the end of the delay alternated on a session by session basis. Experiment 2, therefore, lasted for 120 sessions (in 60 of which, the postevent cue was presented at the start of the delay, and in 60 of which, the postevent cue was presented at the end of the delay).

\section{Results and Discussion}

The data used for the analyses were percentage of correct responses over the last 36 sessions of each condition, separated according to trial type (consistent, inconsistent, neutral, or no-cue), and delay $(2.5,7.5$, or $20.0 \mathrm{sec})$ or serial position of the target stimulus in the list (first, second, 

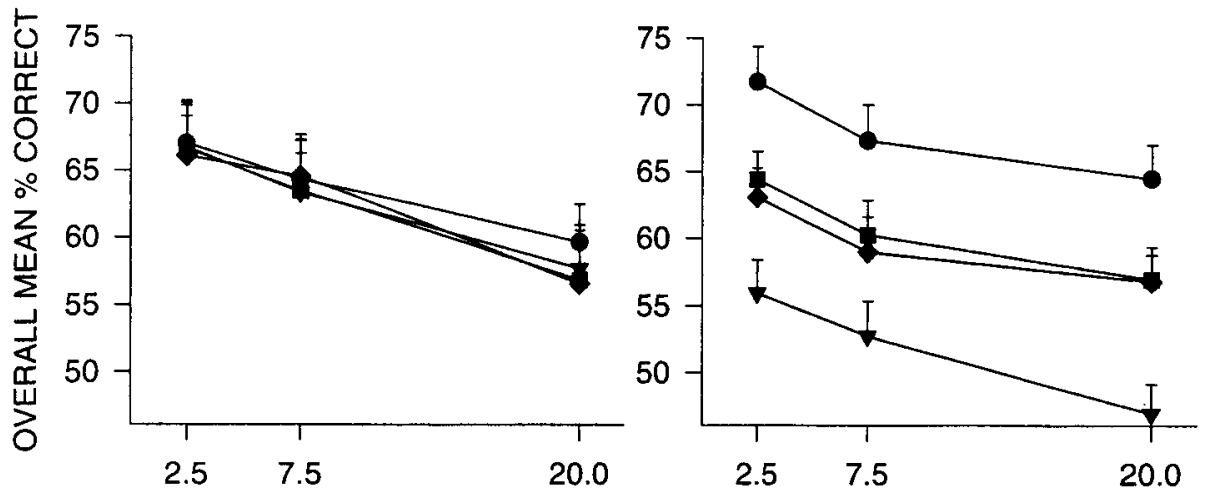

$\operatorname{DELAY}(s)$
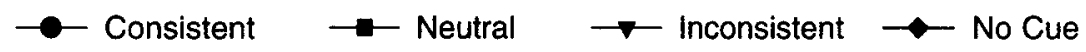

Figure 2. Mean percent correct (averaged across subjects and serial position of the target stimulus) as a function of delay when the postevent cue was presented at the start of the delay interval (left graph) and at the end of the delay (right graph) in Experiment 2. Data are presented separately for consistent (circles), neutral (squares), inconsistent (triangles), and no-cue (diamonds) trial types. Error bars show the standard error associated with each data point.

or third color presented). Figure 2 shows accuracy for each type of trial separated according to delay (collapsed over serial position of the target stimulus). This figure shows that there was no effect of the postevent cue on recognition when it was presented at the start of the delay interval (left graph). In contrast, recognition was strongly biased when the postevent cue occurred at the end of the delay interval (right graph). That is, the subjects were biased toward making recognition responses that matched the color presented as the postevent cue. Consequently, when the postevent cue was the same as the target stimulus (and, therefore, was the same as the correct recognition choice), accuracy was greater than that observed in the neutral or no-cue conditions. On the other hand, when the postevent cue was different from the target stimulus but the same as the incorrect recognition option, accuracy was relatively poorer and typically lower than chance level. Furthermore, there is no obvious difference in performance on neutral trials versus trials in which no postevent cue was presented at all. This biasing effect of postevent cues on recognition performance, dependent on the location of the cue in the delay, is the same as that identified in Experiment 1.

The trends in the data shown in Figure 2 are supported by repeated measures ANOVAs comparing trial type and delay. This analysis revealed a significant main effect of trial type $[F(3,15)=20.4]$ and delay $[F(2,10)=15.2]$ on accuracy when the cue occurred at the end of the delay. The same analysis when the cue was presented at the start of the delay revealed a main effect of delay $[F(2,10)=8.4]$ but no effect of trial type. There was no interaction between trial type and delay, irrespective of whether the cue was presented at the start or the end of the delay. Pairwise comparisons of the different trial types when the cue was presented at the end of the delay indicated that accuracy was lower on the neutral trials than on the consistent trials $[F(1,5)=10.7]$, that accuracy was higher on the neutral trials than on the inconsistent trials $[F(1,5)=30.4]$, and that there was no difference between the no-cue and the neutral trials $[F(1,5)=0.4]$.

Although Experiment 2 replicated the main effect of postevent cues on recognition accuracy shown in Experiment 1 , the two experiments differed in overall levels of accuracy. Generally, although well above chance levels, accuracy was slightly lower in Experiment 2 than in Experiment 1 . For example, summed across all three delays and both conditions, the mean accuracy on the neutral trials was $70 \%$ in Experiment 1, as compared with $62 \%$ in Experiment 2. This difference in overall accuracy levels between the two experiments is not surprising, given that the task used in Experiment 2 would have prevented subjects from employing various mediating behaviors to aid performance (such as positioning themselves in a particular location following a particular color). Furthermore, the target stimulus in Experiment 2 (one target colored light from a sequence of colors) was much less explicit than that in Experiment 1 (a single colored light). Consequently, the procedure in Experiment 2 was more analogous to the standard human situation, in which subjects are not made specifically aware of what stimulus they will be required to remember.

Another difference from the data reported in Experiment 1 was the absence of an interaction between trial type and delay. Such an interaction, as was found in Experiment 1 , is consistent with the idea that a cue's effectiveness is determined by how well the target stimulus can be remembered in the first place. Regardless of whether postevent cues work to interfere with the memory of the original stimulus (Loftus \& Loftus, 1980; Loftus et al., 1978) or whether they bias recognition by being accepted as the correct option when the memory trace for the original target has faded sufficiently (McCloskey \& Zaragoza, 

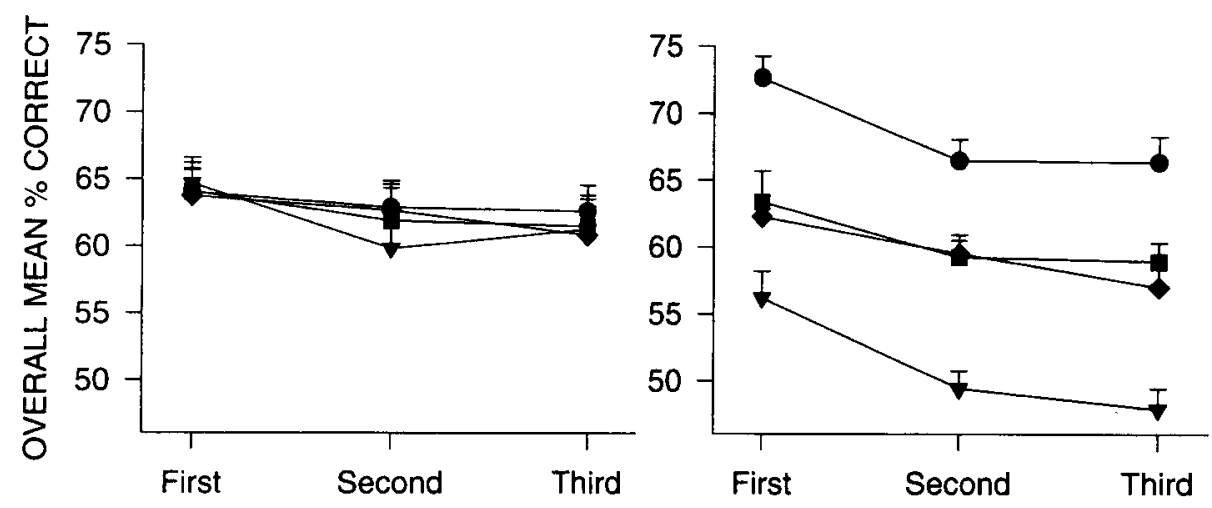

SERIAL POSITION Of TARGET STIMULUS

$\longrightarrow$ Consistent $\rightarrow$ Neutral $\longrightarrow$ Inconsistent $\longrightarrow$ No Cue

\begin{abstract}
Figure 3. Mean percent correct (averaged across subjects and all delays) as a function of the serial position of the target stimulus when the postevent cue was presented at the start of the delay interval (left graph) and at the end of the delay (right graph) in Experiment 2. Data are presented separately for consistent (circles), neutral (squares), inconsistent (triangles), and no-cue (diamonds) trial types. Error bars show the standard error associated with each data point.
\end{abstract}

1985; Zaragoza, McCloskey, \& Jamis, 1987), cues should be less effective at shorter delays than at longer delays. However, the lack of such an interaction in Experiment 2 is likely to have been the result of poorer baseline levels of performance, especially at the shorter delays. Although there was still a significant main effect of delay on accuracy, accuracy was lower overall, and as a result, there was comparatively much less differentiation between performance across delays. Thus, the average decline in accuracy on neutral trials between the shortest and the longest delays was $14.4 \%$ in Experiment 1 versus 8.6\% in Experiment 2. Therefore, if the effectiveness of the postevent cue is determined, entirely or in part, by how well the original stimulus can be remembered, it is not surprising that the cue had a similar effect across different delays in Experiment 2. If the ability to remember the target stimulus is similar across delays, the postevent cue is likely to have a similar extent of effect across those same delays.

Figure 3 shows the same data as those used in Figure 2, except that the data are collapsed across delays and separated according to trial type and serial position of the target stimulus in the list of colors presented at the start of each trial. Consistent with Figure 2, there is a clear biasing effect of the postevent cue when it is presented at the end of the delay interval $[F(3,15)=25.4]$. The relative degree of the cue's impact is the same regardless of whether the target stimulus was the first, middle, or last item to be presented in the list. That is, there was no interaction between serial position of target stimulus and trial type. Figure 3 also shows that there was no effect of postevent cues on target stimuli occurring at any serial position when the cue was presented at the start of the delay interval. In addition to the effects of trial type on accuracy reported above, repeated measures ANOVAs revealed a main effect of serial position of the target stimulus on ac- curacy when the cue occurred at the end of the dela $[F(3,15)=9.6]$, but not when it occurred at the start c the delay $[F(3,15)=0.4]$. Pairwise comparisons of th three serial positions when the cue was presented at th end of the delay indicated that accuracy was higher whe the target stimulus was the first item presented versus th second or the third item $[F(1,5)=8.9$ and $F(1,5)=16$.: respectively], but there was no difference in accuracy the target stimulus was the second versus the third iter presented $[F(1,5)=1.09]$.

The observation in Experiment 2 that target stimu presented at the start of the list are generally more accl rately remembered than items in the middle of the list $i$ consistent with the human and animal data indicating th existence of a serial position effect in memory. A numbe of studies employing different procedures, stimuli, an species have demonstrated that subjects are able to recog nize items occurring at the start or at the end of a list be 1 ter than items occurring in the middle of a list (Glanze \& Cunitz, 1966; Harper, McLean, \& Dalrymple-Alforc 1993; Wright, Santiago, Sands, Kendrick, \& Cook, 1985 Greater accuracy for items early and late in a list are typ ically called primacy and recency effects, respectively. Ir terestingly, although a number of studies with animal have been able to demonstrate the existence of both a pr: macy and a recency effect (e.g., Harper et al., 1993; Wrigh et al., 1985), there was only evidence of a primacy effer in the present study. That is, accuracy was only greate for items occurring early in the list of colors presented $a$ the start of a trial. The absence of a recency effect, how ever, is not surprising, given that the present procedur involved presenting delays and potentially disruptiv postevent cues following presentation of the target iter list. Previous studies examining the serial position effec in humans and animals have shown that relatively shor delays and the presence of disruptive events following lis 
presentation remove the recency effect, while leaving the primacy effect intact (Glanzer, Giantusos, \& Dubin, 1969; Harper et al., 1993; Roediger \& Crowder, 1975).

\section{GENERAL DISCUSSION}

The present research demonstrated that cues presented after a target stimulus could bias DMTS responses in pigeons. Postevent cues biased recognition responses toward the option corresponding to the postevent cue, but only when those cues occurred at the end of the delay interval. When the cue was consistent with the target stimulus (e.g., a red stimulus followed by a red cue), accuracy was greater than that in a neutral condition, whereas, when the cue was inconsistent (the same as the incorrect choice option), accuracy was below chance levels. The biasing effects of postevent cues were observed in two different procedures that differed with respect to how explicit the target stimulus was and mirrored the patterns of effects reported in the human misinformation effect literature.

With respect to previous DMTS research with animals, the present task had features in common with various procedures used to study retroactive and proactive interference effects. Studies on retroactive interference have examined the effect of disruptive events presented during a delay interval, such as free access to food and illuminating the response chamber (Harper \& White, 1997; Jans \& Catania, 1980; Roberts \& Grant, 1978; White, 1985). Consistent with previous research using pigeons and visual stimuli (Roberts \& Grant, 1978), we found that, the later in the delay interval a cue was presented, the greater the effect on accuracy. The focus of the retroactive interference research, however, has been on the effect of novel and, therefore, disruptive events during the delay, whereas the present task used stimuli that were identical to those that had to be remembered. In this manner, the present study was similar to DMTS studies that have examined proactive interference effects arising from stimuli presented or responses made on previous trials. In the proactive interference research, the events that influence performance act to bias recognition toward the response option that is the same as the correct stimulus on the previous trial. Thus, in many respects, the present DMTS paradigm combined features common to both retroactive and proactive interference procedures by using biasing stimuli that are the same as those that have to be remembered (as in the proactive interference research) but presenting them during the delay interval (as in the retroactive interference research). The effects observed with the present procedure were consistent with those observed in both the retroactive and the proactive research but, in addition, expanded on these previous findings by using a task and experimental conditions that were analogous to those used in the human misinformation effect literature.

Although the present research and previous human misinformation studies demonstrate that, when postevent stimuli are the same as target stimuli, performance is above neutral or baseline levels, a study by Grant and Roberts (1976) suggests that, in some circumstances, neu- tral or even familiar postevent cues might impair accuracy. In one experiment, Grant and Roberts initially trained pigeons to match red, green, and blue colors (among a variety of other stimuli). During subsequent testing, using only red and green as sample stimuli and comparisons, they presented either a familiar blue light or a novel (untrained) yellow light during the entire delay interval. Despite being familiar to the subjects, it was found that a blue light presented throughout the delay disrupted overall performance just as much as the entirely novel yellow light presented during the delay. Even though there are several procedural differences between their study and ours (e.g., they presented a colored light throughout the entire delay), these results appear to be at odds with the majority of misinformation findings. A closer look at Grant and Robert's data, however, suggests that the pigeons in their study were biased in a very systematic way by the colored lights presented during the delay. Specifically, when blue was presented during the delay, the pigeons were biased toward choosing the green choice option, whereas when yellow was presented during the delay, the pigeons were biased toward choosing the yellow option. When familiar patterns were used as stimuli interpolated into the delay (as opposed to blue or yellow lights), there was no obvious bias toward choosing either green or red. Therefore, a closer examination of performance in Roberts and Grant's study suggests that colors presented during the delay decreased overall accuracy because of systematic biases to favor one choice alternative over the other in a manner similar to that observed in the present study.

Apart from allowing the assessment of whether postevent cues influenced recognition, the trial conditions used were designed to help in the interpretation of the process by which those cues operate. To this end, the conditions replicated those used in an early study by Loftus et al. (1978), as well as those used in a later study by McCloskey and Zaragoza (1985). Effectively, there were two control conditions. In one control condition, no postevent cues were presented, and in this manner, the task was identical to the standard DMTS task used to examine working memory in pigeons. In the second control condition, a postevent cue was presented that was neutral with respect to either option at the choice phase. McCloskey and Zaragoza have previously argued that this second condition allows assessment of the mechanism by which postevent cues come to bias recognition. One possibility is that the postevent cue interferes with the memory for the original target (misinformation interference in Belli, 1989). Alternatively, the postevent cue may not interfere with memory per se but may come to be accepted as the actual target stimulus (misinformation acceptance in Belli, 1989), especially in situations in which the subject cannot accurately remember the actual target. McCloskey and Zaragoza found no difference in accuracy between trials in which no cue was presented and trials in which a neutral cue was presented. If the postevent cue had operated by interfering with the memory for the actual target, performance on their cued trials should have been im- 
paired, relative to no-cue trials. Thus, they concluded that postevent cues do not operate by interfering with the memory for the original stimulus itself; rather, they operate by being accepted as the correct option. Such a biasing effect is likely to be enhanced as the postevent cue is moved closer to recognition, relative to original target presentation (e.g., by presenting the cue at the end of a relatively long delay). Under such conditions, the original target stimulus is more likely to be forgotten, whereas memory for the postevent cue is relatively strong. Similarly, there was no difference between the no-cue and neutral-cue conditions in the present study, and the biasing effects of consistent and inconsistent cues were at their greatest when presented at the end of the longest delay.

Therefore, the present study has demonstrated that nonhuman animals are affected by misleading postevent cues; moreover, the pattern of misinformation effects is strikingly similar to those found in the human literature. Thus, the literature now shows an intriguing continuity of creatures similarly affected by postevent information: adults, children (see Ceci \& Bruck, 1993, for a review), infants (Rovee-Collier, Adler, \& Borza, 1994), and now pigeons. Future research should heed Rovee-Collier's (1997) suggestion that it is time to simplify our view of the universe when it comes to memory, and we agree.

\section{REFERENCES}

Aggleton, J. P., Keith, A. B., \& Sahgal, A. (1991). The effects of mammillary body and combined amygdala-fornix lesions on tests of delayed non-matching to sample in the rat. Behavioural Brain Research, 40, 145-157.

BELLI, R. F. (1989). Influences of misleading postevent information: Misinformation interference and acceptance. Journal of Experimental Psychologv: Learning, Memory, \& Cognition, 9, 139-145.

Belli, R. F., Windschitl, P. D., McCarthy, T. T., \& Winfrey, S. E. (1992). Detecting memory impairment with a modified test procedure: Manipulating retention interval with centrally presented event items. Journal of Experimental Psychologv: Learning, Memory, \& Cognition, 18, 356-367.

Cecr, S. J., \& Bruck, M. (1993). Suggestibility of the child witness: A historical review and synthesis. Psychological Bulletin, 113, 403439.

EDHousE, W. V., \& WHITE, K. G. (1988). Sources of proactive interference in animal memory. Journal of Experimental Psychology: Animal Behavior Processes, 14, 56-70.

Glanzer, M., \& Cunitz, A. R. (1966). Two storage mechanisms in free recall. Journal of Verbal Learning \& Verbal Behavior, 5, 35I-360.

Glanzer, M., Giantusos, R., \& Dubin, S. (1969). The removal of items from short-term storage. Journal of Verbal Learning \& Verbal Behavior, 8, 435-447.

Grant, D. S., \& RoberTs, W. A. (1976). Sources of retroactive inhibition in pigeon short-term memory. Journal of Experimental Psychology: Animal Behavior Processes, 2, 1-16.
Harper, D. N., Mclean, A. P., \& Dalrymple-Alford, J. C. (1993). List item memory in rats: Effects of delay and delay task. Journal of Experimental Psychology: Animal Behavior Processes, 19, 1-10.

Harper, D. N., McLean, A. P., \& Dalrymple-Alford, J. C. (1994). Forgetting in rats following medial septum or mammillary body damage. Behavioral Neuroscience, 108, 1-12.

HARPER, D. N., \& WhITE, K. G. (1997). Retroactive interference and rate of forgetting in delayed matching-to-sample performance. Animal Learning \& Behavior, 25, 158-164.

JANS, J. E., \& CATANIA, A. C. (1980). Short-term remembering of discriminative stimuli in pigeons. Journal of the Experimental Analysis of Behavior, 34, 177-183.

Kirk, R. C., White, K. G., \& MCNaughton, N. (1988). Low dose scopolamine affects discriminability but not rate of forgetting in delayed conditional discrimination. Psychopharmacology, 96, 541-546.

LofTUS, E. F., \& LoFTUS, G. R. (1980). On the permanence of stored information in the human brain. American Psychologist, 35, 409-420.

LofTus, E. F., Miller, D. G., \& BurNs, H. J. (1978). Semantic integration of verbal information into a visual memory. Journal of Experimental Psychology: Human Learning \& Memory, 4, 19-31.

MaKi, W. S., MOE, J. C., \& BierLey, C. M. (1977). Short-term memory for stimuli, response and reinforcers. Journal of Experimental Psychology: Animal Behavior Processes, 3, 156-177.

MCCARTHy, D., \& Voss, P. (1995). Delayed matching-to-sample performance: Effects of relative reinforcer frequency and of signaled versus unsignaled reinforcer frequencies. Journal of the Experimental Analysis of Behavior, 63, 33-52.

McCloskey, M., \& Zaragoza, M. (1985). Misleading postevent information and memory for events: Arguments and evidence against memory impairment hypotheses. Journal of Experimental Psychology: General, 114, 1-16.

Nevin, J. A., \& Grosch, J. (1990). Effects of signaled reinforcer magnitude on delayed matching-to-sample performance. Journal of Experimental Psychology: Animal Behavior Processes, 16, 298-305.

RoBerTs, W. A., \& GRANT, D. S. (1978). An analysis of light-induced retroactive inhibition in pigeon short-term memory. Journal of $E x$ perimental Psychology: Animal Behavior Processes, 4, 219-236.

RoEDIGER, H. L., III, \& CrowDER, R. G. (1975). The spacing of lists in free recall. Journal of Verbal Learning \& Verbal Behavior, 14, 580602.

Rovee-Collier, C. (1997). Dissociations in infant memory: Rethinking the development of implicit and explicit memory. Psychological Review, 104, 467-498.

Rovee-Collier, C., Adler, S. A., \& Borza, M. A. (1994). Substituting new details for old? Effects of delaying postevent information on infant memory. Memory \& Cognition, 22, 644-656.

WHITE, K. G. ( 1985). Characteristics of forgetting functions in delayed matching to sample. Journal of the Experimental Analysis of Behavior, 44, 15-34.

Wright, A. A., Santiago, H. C., Sands, S. F., Kendrick, D. F., \& CoOK, R. G. (1985). Memory processing of serial lists by pigeons, monkeys and people. Science, 229, 287-289.

Zaragoza, M. S., McCloskey, M., \& Jamis, M. (1987). Misleading postevent information and recall of the original event: Further evidence against the memory impairment hypothesis. Journal of Experimental Psychology: Learning, Memory, \& Cognition, 13, 36-44.

(Manuscript received July 10, 1999;

revision accepted for publication September 9, 1999.) 